\title{
Assessing Correlation between Psychological Resilience and Successful Ageing
}

\author{
Ali Javadpour ${ }^{1,2 *}$, Peter Schofield ${ }^{2}$ and Mohammad Motamedi ${ }^{3}$ \\ ${ }^{1}$ Associate professor in Geriatric Psychiatry, Shiraz Geriatric Research Centre, Shiraz \\ University of Medical Sciences, Iran \\ ${ }^{2}$ Professor in Neuropsychiatry, Research Centre for Brain and Mental Health, Hunter
} Medical Research Institute, University of Newcastle, Australia

Research Article

Volume 3 Issue 2

Received Date: June 08, 2019

Published Date: July 08, 2019

DOI: $10.23880 / \mathrm{mhrij}-16000129$

${ }^{3}$ Master in Clinical Epidemiology, Shiraz Geriatric Research Centre, Shiraz University of Medical Sciences, Iran

*Corresponding author: Ali Javadpour, Associate professor in Geriatric Psychiatry, Department of Psychiatry, Hafez Hospital, Chamran BLV, Shiraz, Iran, Tel: (98) 71 36471939; Email: a.javadpour@gmail.com

\section{Abstract}

Background: The term 'resilience' is applied with reference to a range of biological, psychological and social factors that enable people to survive in a meaningful way in the face of stressful situations and adversities. It is thought that resilience could impact on health status, and high level of resilience is protective against both mental and physical health. It is possible that long term health benefits are influenced by resilience and vice versa. Psychological resilience has been defined as a pervasive and flexible personality trait that helps an individual to cope well into a successful ageing. Successful ageing has been defined as mental, physical and spiritual vigor, into middle age and further years. Successful ageing has also been considered as freedom from disease and disability in the way that people can live independently in later life. Given that resilience represents complex psycho-social behaviors, it could potentially correlate with other gerontology concepts like successful ageing.

Aim: In this study we investigated the correlation between resilience score and some indicators of successful ageing. In current study correlation between psychological resilience score and variables related to successful ageing are examined. Methods: Data was collected in the term one of Olfactory Stress Test longitudinal study at the Hunter Medical Research Institute in Newcastle. Baseline data was collected in 2013-2014 from 290 participant aged 65+ as part of Olfactory Stress Test longitudinal study at the Hunter Medical Research Institute in Newcastle. By using baseline data, we examined the associations between resilience score measured by CD-RISC and some emotional, cognitive and health related variables as indicators of successful ageing.

Result: Initial analyses showed that resilience sore and scores measured mental health related factors such as DASS, CESD, melancholia score, DASS depression and Anxiety subscales were negatively correlated. 


\section{Mental Health \& Human Resilience International Journal}

Correlation analyses between resilience score and items related to cognitive performance revealed that variables such as IQ-code score, PQ-Code Score, MAC-Q, ARCS were negatively correlated and people with higher resilient score reported lower cognitive complaint by both participant and informant. Further analyses also showed that the level of physical activity was positively correlated with resilience score.

There was a negative correlation between number of medical conditions and resilience score $(p=0.013)$. people with more resilient suffering from lower physical illnesses. To check the predictability of mental health related indicators for resilience, emotional and cognitive variables were entered into stepwise regression model. The results showed that depressive symptoms were predictor for lower resilience and more resilience was a predictor for lower cognitive complaint.

Discussion: Our finding is consistent with other studies investigated factors related to resilience in relation to successful ageing. Moreover, finding from this study supported the idea that psychological resilience could be a synonym for Successful ageing and these gerontology concepts could be interrelated in particularly for psychological of successful ageing.

Keywords: Psychological Resilience; Elderly; Successful Ageing

\section{Background}

Psychological resilience has been given attention when psychologists observed that stressors does not always impact childhood development in a negative way and some children even became more strong in face of psychosocial difficulties [1]. Resilience, as an ability to preserve the biological and psychological stability under adversities, has been widely investigated and many studies have tried to provide a clear and assessable definition of resilience. The term 'resilience' is applied with reference to a range of biological, psychological and social factors that enable people to survive in a meaningful way in the face of stressful situations and adversities. It encompasses a comprehensive spectrum of internal factors such as spirituality, caring for self, meaningfulness and external factors like social support, ability to access care and social policy [2].

The current understanding of resilience in some way based on the theories of resilience as a pervasive personality trait; and resilience as an ongoing process. According to personality trait notion, resilience is a pervasive mental resource that enables people to obstacle stressors. In the process model, the level of resilience is based on internal and external factors which adapt the personal response to a particular situation. And resilience could be considered as a moderator between internal personality trait and external risk factors [3-5]. From explanatory view, resilience could be detected if a person cope well with adversities, and in an etiologic process approach, resilience is a process that helps people cope well with stressors. Resilience is considered as a dynamic process varying among people with different psychosocial backgrounds as well as within each individual in different life circumstances [6]. Resilience has been considered as a modifiable psychosocial construct - can be nurtured by intervention- that enhances human capability to adapt in the face of adversity [7].

\section{Resilience in Elderly}

Studies dealing with resilience in old age population commenced around 2000 when positive psychology, intra-individual plasticity and cognitive reserve became the focus of attention, and the first article presenting resilience in elderly was published in $1999[8,9]$.

The aim of studies has been to provide a model for resilience and bring it into practice by examination the catalyzer role of resilience in various age associated changes. They supported the idea that resilience could be a measurable psychological resource with a key role in ageing wellbeing.

Lavretsky in his book 'resilience and ageing' defined resilience as a process that defends against the psychological and physical disturbances caused by 


\section{Mental Health \& Human Resilience International Journal}

adversities and facilitates personal growth and coping. He adopted the Richardson theoretic model for resilience which claims that an individual starts a process to adopt mind, body and spirit to life situation. Disruption in this biopsychosocial stability could lead to either higher level of adaptation or dysfunctional adaptation $[5,10]$.

In a literature review, with the aim to show the concept of psychological resilience in elderly, Fonts and colleagues reviewed 52 international articles. The articles investigated a wide range of bio-psycho-social factors linked to resilience, coping strategies, quality of life and general health in elderly. Factors such as social support, having a job, spirituality, having a partner, generativity, physical health, emotional regulation, self-esteem, behavioral skills, copying strategies, life stories were considered as the precursors of resilience. They concluded that resilience persists throughout the life and they propose that coping is a kind of resistance, management and overcoming of despairing in the face of adversities. In their view an individual needs various biopsychosocial resources such as good health, positive affect, a sense of meaning, spirituality, flexibility, optimism and having an integrated social role to positively cope with adversities [11].

In another review, Kessel and colleagues explored the validity of the current conceptualization of resilience with the aim to define resilience in older people. They categorized them into quantitative (22 papers) and qualitative (20 papers) based on the methods they applied. The quantitative studies used various resilience psychometrics to rate resilience or detect association between resilience and other themes. Kessel pointed that people at their later life are able to implicate individual abilities to overcome stressful life situation. He concluded that resilience in elderly could be enhanced by providing reasonable interventions [2].

Of 94 papers that were collected in these reviews, a few investigated associations between resilience and successful ageing indices. Lamond and colleagues in San Diego used the Connor-Davidson Resilience Scale (CDRISC) to measure resilience in community-dwelling older women. They concluded that a higher score on resilience predictors was strongly and negatively linked to cognitive complaints in older women and higher resilience score was associated with less cognitive complaint [12]. Harris, in a debate on successful aging and resilience, presented two case studies and concluded that resilience could allow people with early stage dementia to continue their lives in a meaningful way [13].
Resilience has also been defined as a pervasive and flexible personality trait that help an individual to cope well into a successful ageing. Resilience has been characterized by five main features: Equanimity (balanced perception of life); meaningfulness (sense of purpose); perseverance (ability to keep going); existtional aloneness (acceptance of one's life); self-resilience (belief in one's capacity).

In this regard resilient individual experience less depression when they migrate, do not succumb to illness, having more self-confidence, are more optimism, able to cope persistently with misfortune [14-16].

\section{Successful Ageing}

Successful ageing has been defined as mental, physical and spiritual vigour, into middle age and further years. successful ageing has also been considered as freedom from disease and disability in the way that people can live independently in later life. As the population is growing and more people live longer, there is increasing curiosity to detect the determinants that help people live longer with better health and quality of life. Factors such as absence of disease, staying physically active, more social engagement have been introduced as indicators of successful ageing [17-20]. So, successful ageing is a multidimensional biopsychosocial concept refers to low risk of illness, higher physical and mental function and more social engagement, life satisfaction and productivity [21-23].

Having a good mental health has also been considered as a component of successful ageing. Some Factors related to resilience such as adaptability, wisdom, optimism, are also potential predictor of successful ageing. And optimistic people as a prototype of resilient individual has been associated with longer life, seeking medical help and caring of himself which are indicators of successful ageing $[16,24]$.

In prior studies characteristics such as physical activity, positive attitude, and enjoy the life have been considered as indicators of successful ageing. In their study Wagnild and colleague's life satisfaction, self-care activities measured as variables related to successful ageing [3].

The main question is whether and how resilience correlated to successful ageing. People who are aging successfully report better health, more social involvement, good mental health which are factors related to resilience. While it is not known that resilience 


\section{Mental Health \& Human Resilience International Journal}

as a cause and effect of successful ageing but it appears they are interrelated. According to Rowe and Kahn study resilience will facilitate successful ageing [25].

Good mental is a fundamental factor for both resilience and successful ageing. And incorporate information on mental health has been suggested to be considered in definition of successful ageing and negative emotional experience like depression seems to be negatively related to successful ageing [26,27]. Result from cognitive studies strongly supported the intellectual functioning with longevity and successful ageing [28]. Some emotional state and personality has been reported as predictor of successful ageing and neuroticism negatively associated with ageing successfully. Likewise positive emotional state, xtroversion have been reported as predictors of successful ageing [29].

While successful ageing is an arguing core concept in gerontology but definition has been widely argued. In conclusion; health condition, good physical activity, high cognitive performance, positive emotions, and social involvement are the most criteria accepted as indicators of successful ageing [30].

\section{Logic for Study}

Given that resilience represents complex psycho-social behaviors, it could potentially correlate with other gerontology concepts like successful ageing. It is thought that resilience could impact on health status, and high level of resilience is protective against both mental and physical health [31]. It is possible that long term health benefits are influenced by resilience and vice versa. Rak proposed a clinical usage for resilience intervention model that explores resilience qualities in individuals and nurture them [7].

There is certain logic to investigate the correlation between resilience scale and indicators of successful ageing. While there has been great trend toward studying resilience in the elderly over two recent decades, the association between resilience and aspects of successful ageing in the old age population has not adequately investigated. Resilience in old age depends on emotional and cognitive reserve, and there is an assumption that resilience increases in old age and builds up a successful adaptation to adversities [32]. We designed this study to examine the relationship between resilience score assessed by a valid resilience instrument factors that are considered as indicators for successful ageing. The question is whether resilience score is a predictor for SA in people aged over 65 .

\section{Aim}

In current study correlation between psychological resilience score and variables related to successful ageing are examined. The sub-objectives of this study are:

- To investigate the association of resilience scores measured by the CD-RISC and emotional symptoms in older people.

- To examine the predictive value of resilience for cognitive complain and performance in older persons.

- To examine the association between resilience and daily activities in older persons

- To examine the association between resilience and some health related factors in older persons.

\section{Expected Outcomes, Significance or Rationale}

Considering that resilience may be both influenced by and itself impact biological, psychological, and social factors, an individual measure of this construct may well be associated with factors identified as determinants of successful ageing. Indeed, we hypothesize that resilience score will be associated both with indices of successful ageing in elderly, cross-sectional and longitudinally. The results of this study would have potential utility in both clinical practices and research, especially if, as is being investigated in other studies, resilience could be taught.

\section{Methodology}

Data was collected in the term one of Olfactory Stress Test longitudinal study at the Hunter Medical Research Institute in Newcastle. Baseline data was collected in 2013-2014 from 290 participant aged 65+ as part of Olfactory Stress Test longitudinal study at the Hunter Medical Research Institute in Newcastle. By using baseline data, we will first examine the associations between resilience score measured by CD-RISC and some emotional, cognitive and health related variables as indicators of successful ageing.

\section{Measures}

Resilience is measured by Connor-Davidson resilience scale (CD-RISC) [31]. Cognitive functioning is measured by Mini mental status examination (MMSE). The MMSE is a quantitative assessment of cognitive function. It gives a brief assessment of the person's orientation, recall ability, memory, and language ability [32,33]. Audio Recorded Cognitive Screen (ARCS) was uses as a screening cognitive measure. ARCS is administered through headphones via an audio device, and subjects respond by writing their answers in a booklet for later scoring [34-36]. MAC-Q is a 


\section{Mental Health \& Human Resilience International Journal}

self-report questionnaire of five questions meeting diagnostic criteria for age-associated memory impairment (AAMI [37].

Informant questionnaire(IQ-Code) and the Participant Questionnaire on Cognitive Decline in the Elderly (PQCode) ask specific questions about memory complaints form the informant and participants separately [38]. Depression anxiety and stress scale (DASS) were used to score emotional related symptoms. The DASS21 is a set of three self-report scales measuring negative emotional states such as depression, anxiety and stress [39]. Depressive symptoms were specifically measured by using CES-D [40]. Moreover, data were collected by using SDS (Severe Depressive symptoms) and melancholia items are considered as indicator for depressive symptoms as well. These two survey instruments asked participants how they feel in different circumstances in their current life.

To check the level of physical activity the FAQ was used. The Functional Activities Questionnaire is a 10 item instrument that asks the participant if they have any difficulty or need help with specific tasks [41]. The number of medical conditions reported by the participants; collected in health assessment clinics; were considered as indicators of physical health in the participants.

\section{Results}

\section{Data Aanalyses}

Spss version 23 was used to analyses data.to show normal distribution of resilience score was confirmed by using Kolmgreve -Sminnove test and $\mathrm{Q}-\mathrm{Q}$ plot chart. Independent $\mathrm{t}$-test and one way analyses were used to compare means of resilience score among categorical variables. The correlation between numeral variables was assessed by using Pearson correlation coefficient and Eta was employed to assess correlation between categorical variables like history of treatment for depression. In the next step we used multiple regression analyses to check the predictability of various items for resilience score.

Sociodemographic of sample size is presented in table 1.

Initial analyses showed that resilience sore and scores measured mental health related factors such as DASS, CES-D, melancholia score, DASS depression and Anxiety subscales were negatively correlated. Eta analyses showed higher resilience score among people without

Ali Javadpour, et al. Assessing Correlation between Psychological Resilience and Successful Ageing. Ment Health Hum Resilience Int J 2019, 3(2): 000129. history of treatment for depression than individuals who had history of treatment for depression $(\mathrm{p}=0.004)$.

\begin{tabular}{|c|c|}
\hline Variable & No. (\%) \\
\hline Male & $167(56.6)$ \\
\hline Female & $128(43.4)$ \\
\hline $60-69.9$ & $107(36.3)$ \\
\hline $70-79.9$ & $144(48.8)$ \\
\hline 80 & $41(13.9)$ \\
\hline \multicolumn{2}{|c|}{ Education } \\
\hline High school not completed & $14(4.7)$ \\
\hline Junior high school & $93(31.5)$ \\
\hline Senior high school or TAFE & $114(38.6)$ \\
\hline University & $74(25.1)$ \\
\hline Employment Status \\
\hline Yes & $28(9.5)$ \\
\hline No & $264(89.5)$ \\
\hline Retirement Status \\
\hline Yes & $272(92.2)$ \\
\hline No & $21(7.1)$ \\
\hline single & $12(4.1)$ \\
\hline married & $19(72.5)$ \\
\hline divorced & $2(0.4)$ \\
\hline separated & $61(13.9)$ \\
\hline divorced & $6(2)$ \\
\hline cohabiting & \\
\hline
\end{tabular}

Table1: Sociodemographics of sample study.

\begin{tabular}{|c|c|}
\hline Variable & Res Score (r) \\
\hline DASS depression & $-0.265^{*}$ \\
\hline melancholia & $-0.225^{*}$ \\
\hline CESD & $-0.241^{*}$ \\
\hline DASS21 & $-0.298^{*}$ \\
\hline Sleep total & -0.057 \\
\hline IQ Code Score & $-0.105^{*}$ \\
\hline FAQ Average & $-0.171^{*}$ \\
\hline MAC-Q & $-0.264^{*}$ \\
\hline ARCS overall score & $0.106^{*}$ \\
\hline Intracranial Vol (mm^3) & -0.088 \\
\hline PQCODE & $-0.193^{*}$ \\
\hline Physical Activity & $0.109^{*}$ \\
\hline DASS anxiety & $-0.148^{*}$ \\
\hline
\end{tabular}

Table 2: Correlation Coefficients between resilience score and study variables.

Correlation analyses between resilience score and items related to cognitive performance revealed that 


\section{Mental Health \& Human Resilience International Journal}

variables such as IQ-code score, PQ-Code Score, MAC-Q, ARCS were negatively correlated and people with higher resilient score reported lower cognitive complaint by both participant and informant. Further analyses also showed that the level of physical activity was positively correlated with resilience score.

To check another determinant of successful ageing the relation between resilience score and the number of medical condition reported by the participant was examined There was a negative correlation between number of medical conditions and resilience $\mathrm{score}(\mathrm{p}=0.013)$. People with more resilient suffering from lower physical illnesses.

To check the predictability of mental health related indicators for resilience, emotional and cognitive variables including DASS, DASS Anxiety, DASS Depression, CES-D, Melancholia, were entered into stepwise regression model. The results showed that depressive symptoms were predictor for lower resilience (Table 3).

\begin{tabular}{|c|c|c|c|c|c|}
\hline Variable & B & SE & R & $\mathbf{R}^{\mathbf{2}}$ & P Value \\
\hline constant & 80.749 & 1.01 & & & \\
\hline DASSdepression & 0.88 & 0.119 & 0.275 & 0.075 & $0.000^{*}$ \\
\hline Melancholia & -0.889 & 0.386 & 0.308 & 0.095 & $0.000^{*}$ \\
\hline
\end{tabular}

$*_{\alpha}=0.05$

Table 3: Multiple regression analyses for depression.

Furthermore the scores of measures assessed cognitive complaints and performance including IQ-Code, PQ-Code, MAC-Q, ACRCS were entered into stepwise regression model and the results showed that resilience was a predictor for lower cognitive complaint (Table 4).

\begin{tabular}{|c|c|c|c|c|c|}
\hline Variable & B & SE & R & $\mathbf{R}^{\mathbf{2}}$ & P Value \\
\hline constant & 134.987 & 9.798 & & & \\
\hline MAC-Q & -0.767 & 0.0209 & 0.296 & 0.088 & $0.000^{*}$ \\
\hline PQCODE & -0.351 & 0.128 & 0.367 & 0.135 & 0 \\
\hline FAQ Average & -1.279 & 0.589 & 0.386 & 0.149 & 0.004 \\
\hline
\end{tabular}

$*_{\alpha}=0.05$

Table 4: Multiple regression analyses for cognitive measures.

\section{Discussion}

Resilience has also been defined as a pervasive and flexible personality trait that help an individual to cope well into a successful ageing. In this study we investigated the correlation between resilience score and some indicators of successful ageing. Our finding is consistent with other studies investigated factors related to resilience in relation to successful ageing [16,24-29].

In present study variables related to mental health such as depressive symptoms, anxiety, level of stress was negatively associated with resilience and more resilient individuals reported lower negative emotional symptoms. In some studies, having a good mental health has also been considered as a component of successful ageing. Some Factors related to resilience such as adaptability, wisdom, optimism, are also potential predictor of successful ageing. And optimistic people as a prototype of resilient individual has been associated with longer life, seeking medical help and caring of himself which are indicators of successful ageing $[16,24]$. Good mental is a fundamental factor for both resilience and successful ageing. And incorporate information on mental health has been suggested to be considered in definition of successful ageing [26]. Negative emotional experience like depression seems to be negatively related to successful ageing [27]. Some emotional state and personality has been reported as predictor of successful ageing and neuroticism negatively associated with ageing successfully. Likewise positive emotional state, extroversion have been reported as predictors of successful ageing [29]. In this regard resilient individual experience less depression when they migrate, do not succumb to illness, having more self-confidence, are more optimism, able to cope persistently with misfortune [14$16]$.

Consistent with other studies in this study factors related to cognitive performance such reported cognitive 


\section{Mental Health \& Human Resilience International Journal}

performance and complaint by either patients or their informant were negatively correlated with the level of resilient; so that more resilient individuals less likely complained of their cognitive performance. Lamond and colleagues in San Diego used the Connor-Davidson Resilience Scale (CD-RISC) to measure resilience in community-dwelling older women. They concluded that a higher score on resilience predictors was strongly and negatively linked to cognitive complaints in older women and higher resilience score was associated with less cognitive complaint [12]. Harris, in a debate on successful aging and resilience, presented two case studies and concluded that resilience could allow people with early stage dementia to continue their lives in a meaningful way [13]. Result from cognitive studies strongly supported the intellectual functioning with longevity and successful ageing [28].

In this study we searched relationship of some more indicators of successful ageing like physical activity, physical health in relation to psychological resilience. More resilient People were more physically active and reported less physical illnesses. In line with our findings some prior studies characteristics such as physical activity, positive attitude, and enjoy the life have been considered as indicators of successful ageing. In their study Wagnild and colleague's life satisfaction, self-care activities measured as variables related to successful ageing [3]. People who are aging successfully report better health, more social involvement, good mental health which is factors related to resilience. According to Rowe and Kahn study resilience will facilitate successful ageing [25]. People with more resilient suffering from lower physical illnesses.

The main question is whether and how resilience correlated to successful ageing. People who are aging successfully report better health, more social involvement, good mental health which are factors related to resilience. While it is not known that resilience as a cause and effect of successful ageing but it appears they are interrelated. According to Rowe and Kahn study resilience will facilitate successful ageing [25]. Row and colleagues in their study found that resilience will facilitate successful ageing [25].

Moreover, finding from this study supported the idea that psychological resilience could be a synonym for Successful ageing and these gerontology concepts could be interrelated in particularly for psychological of successful ageing. As this study was not specifically designed to study association between resilience and successful ageing, further study using measures defining successful ageing might be needed is recommended.

\section{References}

1. Rutter M (2013) Annual Research Review: Resilience-clinical implications. J Child Psychol Psychiatry 54(4): 474-487.

2. van Kessel G (2013) The ability of older people to overcome adversity: a review of the resilience concept. Geriatr Nurs 34(2): 122-127.

3. Wagnild G (2003) Resilience and successful aging. Comparison among low and high income older adults. Journal of Gerontological Nursing 29(12): 42-49.

4. Leipold B, Greve B (2009) Resilience, a conceptual Bridge between copying and developement. european psychologist 14(1): 40-50.

5. Lavertsky H (2014) Resilience and ageing; research and practice. John Hopkins University Press, Volume 1.

6. Hardy SE, Concato J, Gill TM (2004) Resilience of community-dwelling older persons. J Am Geriatr Soc 52(2): 257-262.

7. Rak C (2002) Heroes in the nursery. Journal of clinical psychology 58(3): 247-260.

8. Bergeman CS, Wallace KA (1998) Resilience in later life, in life -span perspective on health and illness. In: Whitman TL, Merluzzi TV, White RD, Psychology press, USA, pp: 207-225.

9. Seligman ME, Csikszentmihalyi M (2000) Positive psychology. An introduction. Am Psychol 55(1): 5-14.

10. Richardson GE (2002) The metatheory of resilience and resiliency. J Clin Psychol 58(3): 307-321.

11. Fontes AP, Neri AL (2015) Resilience in aging: literature review. Cien Saude Colet 20(5): 1475-1495.

12. Lamond AJ, Depp CA, Allison M, Langer R, Reichstadt $\mathrm{J}$, et al. (2008) Measurement and predictors of resilience among community-dwelling older women. J Psychiatr Res 43(2): 148-154.

13. Harris PB (2008) Another wrinkle in the debate about successful aging: the undervalued concept of 


\section{Mental Health \& Human Resilience International Journal}

resilience and the lived experience of dementia. Int J Aging Hum Dev 67(1): 43-61.

14. Caplan G (1990) Loss, stress and mental health. Community Mental Health Journal 26(1): 7-48.

15. Beardslee WR (1989) The role of self-undestanding in resilient individuals: The developement of a perspective. American Journal of Orthopsychiatry 59(2): 266-178.

16. Druss RG, Douglas CJ (1988) Adaptive response to illness and disability: Healthy denial. General Hospital Psychiatry 10(3): 163-168.

17. Rowe JW, Kahn RL (1997) Successful ageing. Gerontologist 37(4): 433-440.

18. Ruuskanen JM, Rouppila I (1995) Physical activity and psychological wellbeing among people aged 65 to 84 years. Age and Ageing 24(4): 292-296.

19. Unger JB, Johnson CA, Marks G (1997) Functional decline in the elderly: Evidence for direct and stressbuffering protective effects of social interactions and physical activity. Annals of behavioural Medicine 19(2): 152-160.

20. Strawbridge WJ, Cohen RD, Shema SJ, Kaplan GA (1996) Successful ageing: predictors and associated activities. American Journal of epidemiology 144(2): 135-141.

21. Rowe JW, Kahn RL (1987) Human ageing: usual and successful. Science 237(4811): 143-149.

22. Lehr U (1982) Socio-psychological correlates of longevity. Annual Review of Gerontology and Geriatrics 3: 102-147.

23. Siegrist J, Knesebeck O, Pllack CE (2004) Social productivity and well-being of older people. A sociological explanation. Social Theory and Health 2(1): 243-246.

24. Maruta T, Colligan RC, Malinchoc M, Offord KP (2000) Optimists vs pesimists: survival are among medical patients over a 30-year period. Mayo Clinic Proceedings 75(2): 140-143.

25. Rowe JW, Kahn RL (2000) Successful ageing and disease prevention. Advances in Renal Replacement Therapy 7(1): 70-77.

Ali Javadpour, et al. Assessing Correlation between Psychological Resilience and Successful Ageing. Ment Health Hum Resilience Int J 2019, 3(2): 000129.
26. Kok AA, Aartsen MJ, Deeg DJ, Huisman M (2017) Capturing the diversity of successful ageing: an operational definition based on 16-year trajectories of functioning. Gerontologist 57(2): 240-251.

27. Jeste DV, Savla GN, Thompson WK, Vahia IV, Glorioso DK, et al. (2013) Association between older age and more successful aging: critical role of resilience and depression. American Journal of Psychiatry 170(2): 188-196.

28. Batty GD, Wennerstad KM, Smith GD, Gunnell D, Deary IJ, et al. (2009) IQ in Early adulthood and Mortality by Middle Age: cohort study of 1 million Swedish men. Epidemiology 20(1): 100-109.

29. Fernández-Ballesteros R, Zamarrón MD, Rudinger G, Schroots JJ, Hekkinnen E, et al. (2004) Assessing Competence. The European Survey on Ageing Protocol. Gerontology 50(5): 330-347.

30. Fernández-Ballesteros R, García LF, Abarca D, Blanc L, Efklides A, et al. (2008) Lay concept of ageing well: cross-cultural comparisons. Journal of the American Geriatric Society 56: 950-952.

31. Dong F, Nelson C, Shah-Haque S (2013) A modified CD-RISC: including previously unaccounted for resilience variables. Kansas Journal of Medicine 6(1): 11-20.

32. Staudinger UM, Marsiske M, Baltes PB (1993) Resilience and levels of reserve capacity in later adulthood: perspective from life-span theory. Development and psychopathology 5(4): 541-566.

33. Folstein MF, Folstein SE, McHugh PR (1975) "Minimental state". A practical method for grading the cognitive state of patients for the clinician. J Psychiatr Res 12(3): 189-198.

34. Lechner-Scott J, Kerr T, Spencer B, Agland S, Lydon A, et al. (2010) The Audio Recorded Cognitive Screen (ARCS) in patients with multiple sclerosis: a practical tool for multiple sclerosis clinics. Mult Scler 16(9): 1126-1133.

35. Loughland CM, Allen J, Gianacas L, Schofield PW, Lewin TJ, et al. (2010) Brief neuropsychological profiles in psychosis: a pilot study using the Audio Recorded Cognitive Screen (ARCS). Acta Neuropsychiatrica 22(5): 243-252. 


\section{Mental Health \& Human Resilience International Journal}

36. Schofield PW, Stephen J Lee, Terry J Lewin, Grant Lyall, Jonson Moyle, et al. (2010) The Audio Recorded Cognitive Screen (ARCS): a flexible hybrid cognitive test instrument. Journal of Neurology, Neurosurgery and Psychiatry 81(6): 602-607.

37. Crook TH, Feher EP, Larrabee GJ (1992) Assessment of memory complaint in age-associated memory impairment: the MAC-Q. International Psychogeriatrics 4(2): 165-176.

38. Gavett R, Dunn JE, Stoddard A, Harty B, Weintraub S (2011) The Cognitive Change in Women Study (CCW): Informant Ratings of Cognitive Change but not Self Ratings are Associated with Neuropsychological Performance Over Three Years. Alzheimer disease and associated disorders 25(4): 305-311.
39. Henry JD, Crawford JR (2005) The short-form version of the Depression Anxiety Stress Scales (DASS-21): Construct validity and normative data in a large non-clinical sample. British Journal of Clinical Psychology 44(2): 227-239.

40. Radloff LS (1977) The CES-D scale a self-report depression scale for research in general population. Applied Psychological Measurement 1(3): 385-401.

41. Pfeffer RI, Kurosaki TT, Harrah CH, Chance JM, Filos S (1982) Measurement of Functional Activities in Older Adults in the Community. Journal of Gerontology 37(3): 323-329. 